\title{
Polarization Reconstruction of Cosmic Rays with the ARIANNA Neutrino Radio Detector
}

\section{Leshan Zhao $^{a, *}$ on behalf of the ARIANNA Collaboration}

(a complete list of authors can be found at the end of the proceedings)

${ }^{a}$ University of California, Irvine

Irvine, USA

E-mail: leshanz@uci.edu

The ARIANNA detector is designed to detect neutrinos of energies above $10^{16} \mathrm{eV}$. Due to the similarities in generated radio signals, cosmic rays are often used as test beams for neutrino detectors. Some ARIANNA detector stations are equipped with antennas capable of detecting air showers. The radio emission properties of air showers are well understood, and the polarization of the radio signal can be predicted from arrival direction with high precision. For this reason, cosmic rays can be used as a proxy to assess the reconstruction capabilities of the ARIANNA neutrino detector. We report on dedicated efforts of reconstructing the polarization of cosmic-ray radio pulses. A total of 148 cosmic rays are identified from over 90,000 triggered events collected between Nov 21, 2018 and Mar 15, 2019. A cut was put on these events requiring them to have a signal-to-noise (SNR) ratio of at least 4.5 in all upward-facing channels. This was to improve the performance of arrival direction and polarization reconstruction algorithms. Polarization of these cosmic rays were reconstructed with a resolution of 3.5 degrees (68\% containment), which agrees with the expected value we obtained from simulation. Furthermore, if we subselect events with zenith angle greater than $70 \mathrm{deg}$, the contribution to polarization of Askaryan effect is reduced, which reduces the error in the predicted polarization. With limited statistics, we find the polarization uncertainty is reduced to $1.3 \mathrm{deg}$ ( $68 \%$ containment).

$37^{\text {th }}$ International Cosmic Ray Conference (ICRC 2021)

July 12th-23rd, 2021

Online - Berlin, Germany

\footnotetext{
${ }^{*}$ Presenter
} 


\section{Introduction}

The ARIANNA experiment is located on Ross-Ice-Shelf in Antarctica. In a uniquely radio quiet area, a hexagonal array of pilot-stations has been taking data for several years and a limit on the high-energy neutrino flux has been derived [1]. In addition to downward-facing log-periodic dipole antennas (LPDA) and dipole antennas that are designed for in-ice shower detection, some of the detector stations have been equipped with upward-facing LPDAs that are optimized for air shower detection. As the spacing between stations is so large, air-shower characteristics have to be reconstructed from a single station as opposed to what typical air-shower radio arrays are using [2-4].

For neutrino signals, polarization direction is important because it is needed to reconstruct neutrino arrival direction [5]. Studies show that polarization is the dominant uncertainty on the neutrino direction [5-7]. The other parameters that impact the neutrino direction, namely signal arrival direction and viewing angle, can typically be measured more precisely. Thus, an accurate reconstruction of the signal polarization is the key to improve the angular resolution.

The frequency content and duration of the radio pulse generated by cosmic rays is similar to what is expected for neutrinos, and since the LPDAs are buried in snow, the local effects on antenna response is included in a similar way. This makes the measurement of polarization of cosmic rays a useful tool to certify the expected performance of the ARIANNA stations. Another useful feature of cosmic ray polarization is that it is predominantly polarized horizontally for most cosmic-ray directions and can already be predicted with good precision from the air-shower direction, unlike neutrinos where the polarization direction is unknown a priori. This unique feature makes diagnoses on reconstruction easier.

\section{Cosmic rays identification with ARIANNA}

The detector station used in this study is equipped with 4 upward-facing LPDAs, 2 downwardfacing LPDAs, and 2 dipole antennas. The 4 upward-facing LPDAs consist of 2 pairs of parallel LPDAs with the 2 pairs orthogonal to each other. The trigger requires the waveform voltage to exceed a high and a low threshold within $5 \mathrm{~ns}$ on one upward-facing channel, and again on another upward-facing channel within $32 \mathrm{~ns}$ for a two of four majority logic requirement [2]. Cosmic ray identification criteria are designed basing on spatial and temporal distribution of the events, correlation with simulated events, signal amplitude, and the difference in correlation between parallel channels. The cuts discussed below are applied to data in the sequence in which they are discussed.

\subsection{Overview of data}

In this article we concentrate on data taken between November 22th 2018 and the end of data taking at March 15th 2019. 98039 events are triggered during this period of time with the triggering logic discussed above.

\subsection{Correlation analysis based on simulations}

Simulation studies discussed in this paper are based on a set of simulated events generated with CoREAS. CoREAS is a Monte Carlo code for the simulation of radio emission from extensive air 


\begin{tabular}{||c|c|c||}
\hline Cut name & Number of events remaining(data) & Cut efficiency(simulation) \\
\hline rate cut & 33884 & N/A \\
\hline corr cut & 298 & 0.97 \\
\hline parallel cut & 218 & 1.00 \\
\hline downward cut & 206 & 0.99 \\
\hline dipole cut & 192 & 0.99 \\
\hline direction cut & 187 & 0.99 \\
\hline zenith cut & $148(0.79)$ & 1.00 \\
\hline SNR cut & $27(0.18)$ & 0.12 \\
\hline
\end{tabular}

Table 1: Table of cuts applied in cosmic rays identification process. Cut efficiency in the third column is calculated from simulation. It is defined as the number of simulated cosmic rays after the cut divided by the number of cosmic rays before the cut.

showers [8]. The simulated data set is a large library consisting of 1000 showers with 160 observers in each distributed over a star-shape layout that efficiently samples the radio footprint. Those simulated events cover a large range of different arrival directions and energies. After correcting for amplifier response, measured voltage traces from the four upward facing LPDAs are correlated with the set of simulated events and a normalized correlation coefficient is calculated for each channel. The average correlation coefficients over the 1000 simulated events is used in the study. The largest correlation of the four upward-facing channels is defined to be the correlation of the event.

\section{3 trigger rate cut (rate cut)}

Cosmic rays are expected to be distributed randomly in time. On the other hand, man-made signals and wind-induced events tend to cluster in time. Therefore, removing temporally clustered events would highly improve the purity of the data without losing many cosmic rays. The trigger rate cut is designed so that temporally clustered events with large correlations are removed. We only focus on events with large correlation in this cut because low correlation events caused by thermal fluctuation are almost always temporally clustered and they can be easily removed in the next steps. The cut logic is that, within each 30 min long data-taking window, if the number of events whose correlation is larger than 0.4 is larger than 5 , all events taken from this window are removed. This number corresponds to a $2.8 \times 10^{-3} \mathrm{~Hz}$ trigger rate. 33884 events pass this cut. The dead-time due to this trigger rate cut is 40,350 minutes, which corresponds to $29 \%$ of the total live-time of the season. We show the correlation for all events in Fig. 1.

\section{4 correlation cut (corr cut)}

Over $99 \%$ of the events passing the trigger rate cut are low-amplitude events that are triggered due to random thermal fluctuations. Due to their thermal noise nature, these events can be easily identified by correlating their waveform to the simulated waveform of cosmic rays. A correlation larger than 0.5 is needed to pass this cut. As a result, 298 events pass this cut, which corresponds to $0.9 \%$ of the events passing the previous cut. 


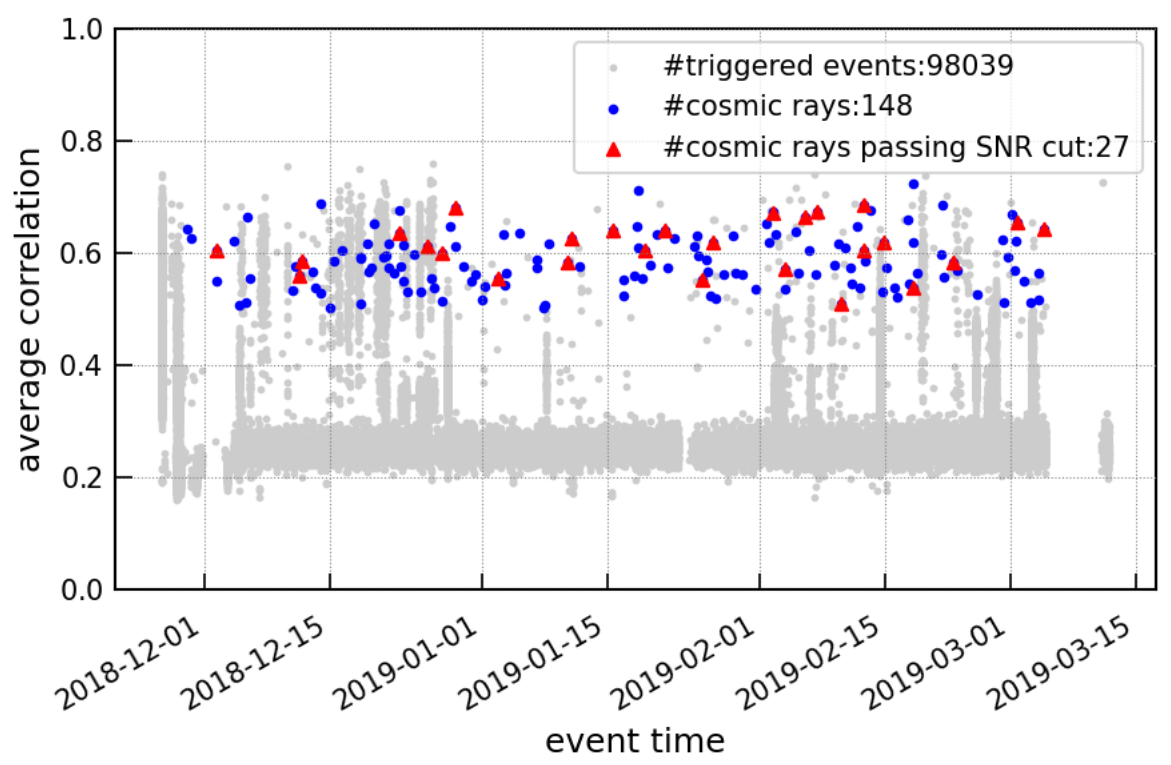

Figure 1: Temporal distribution of cosmic ray events. Dots in grey are triggered events. Dots in blue are cosmic ray events. Red triangles are cosmic rays with SNR $>4.5$ in all upward channels.

\section{5 parallel channel correlation cut (parallel cut)}

Due to the plane wave nature of air showers and the geometry of the LPDAs, cosmic ray events are expected to produce similar signals in parallel LPDA channels. The parallel channel correlation cut is designed to remove events in which the difference in correlation between 2 parallel channels is greater than 0.15. As discussed above, the station is equipped with 2 pairs of parallel LPDAs. If either of the pairs fails this criterion, the event is removed. Out of the 298 events passing the previous cut, 218 events pass this cut.

\subsection{Downward-facing LPDAs cut (downward cut)}

According to simulation study, there is a correlation between signal amplitude in upward and downward facing LPDAs. The ratio in amplitude varies from 0.3 to 0.6 depending on signal arrival direction. On the other hand, this ratio is larger for many background events. This cut removes events in which the ratio between the largest amplitude in two downward channels and the largest amplitude in the four upward channels is larger than 0.7. 206 events pass this cut.

\subsection{Dipole antenna cut (dipole cut)}

Due to their nearly horizontal polarization, cosmic rays generate virtually no signals in dipole antennas. On the other hand, noise from electronics and other man-made sources can generate relatively large signals in dipole antennas. This cut is designed so that events that has a correlation coefficient larger than 0.32 in any of the two dipole antennas are removed. 192 events pass this cut

\subsection{Signal arrival direction cut (direction cut)}

For events passing the above 5 cuts, the signal arrival direction is reconstructed basing on relative signal time delay and cross-correlation between upward-facing channels [7, 9]. According 
to simulation study, the reconstruction resolution is 1.6 degree in azimuth and 1.3 degree in zenith (68\% containment). Considering that cosmic rays are expected to be distributed randomly in space and that the number of cosmic-ray candidates passing previous cuts is low, it is unlikely that multiple cosmic rays come from the same direction. Therefore, the signal arrival direction cut is made so if there are more than 3 events clustered within $3^{\circ}$ in zenith or azimuth, all these events are removed. As a result, 187 events pass this cut.

\subsection{Zenith cut (zenith cut)}

We further require events to have a reconstructed arrival direction in zenith to be larger than $40^{\circ}$. This is because we found that many non-cosmic ray events have a small zenith angle. In fact, many typical background events, wind-induced events and signals from overhead air crafts for example, reconstruct to small zenith angles. On the other hand, the expected number of cosmic rays coming from small zenith angles is small due to the small solid angle and the reduced detection efficiency. Therefore, this cut is expected to reject a large fraction of background events while keeping most cosmic rays. 148 events pass this cut.

Events passing the above 7 cuts are used in the following analysis.

\section{Polarization reconstruction}

In order to reconstruction the polarization of an air shower, the electric field needs to be reconstructed. In this study we use the forward-folding technique to reconstruct the electric field. Instead of recovering numerically the incident electric field frequency bin by frequency bin, the forward-folding technique fits an analytic model of the electric-field pulse directly to the measured voltages in the time domain. This technique is found to have significantly better accuracy compared to standard unfolding methods, especially for small signal-to-noise ratio ${ }^{1}$ (SNR) events [9]. As shown in Fig. 2, the measured voltage traces agree with the analytic solution of the forward-folding technique to high precision. The agreement is particularly good for low frequency components.

The polarization angle is defined to be the angle between $\mathbf{e}_{\phi}$ and $\mathbf{e}_{\theta}$ components of the electric field vector. We calculate the polarization using $\arctan \left(\sqrt{E_{\phi}} / \sqrt{E_{\theta}}\right)$, where $E_{\phi}$ and $E_{\theta}$ are the energy fluence of $\phi$ and $\theta$ components of the reconstructed electric field, respectively. Here we make an additional cut which requires all upward-facing channels to have an SNR larger than 4.5. This cut is needed to ensure a precise arrival direction reconstruction, which is needed to predict the signal polarization. In this work, we prioritize a high cosmic-ray purity with a reliable direction reconstruction to be able to make a reliable assessment of the polarization reconstruction capabilities. This cut selects 27 events out of the 148 events data set.

\subsection{Calculation of predicted polarization}

In order to evaluate the performance of the reconstruction, a prediction of polarization is needed. The polarization error is defined to be the absolute value of the difference between the predicted and reconstructed polarization vector. In Antarctica where the local geomagnetic field is virtually vertical, polarization of air showers is dominated by geomagnetic effect with a small

\footnotetext{
${ }^{1} \mathrm{SNR}$ is defined as the maximum amplitude divided by the noise RMS
} 

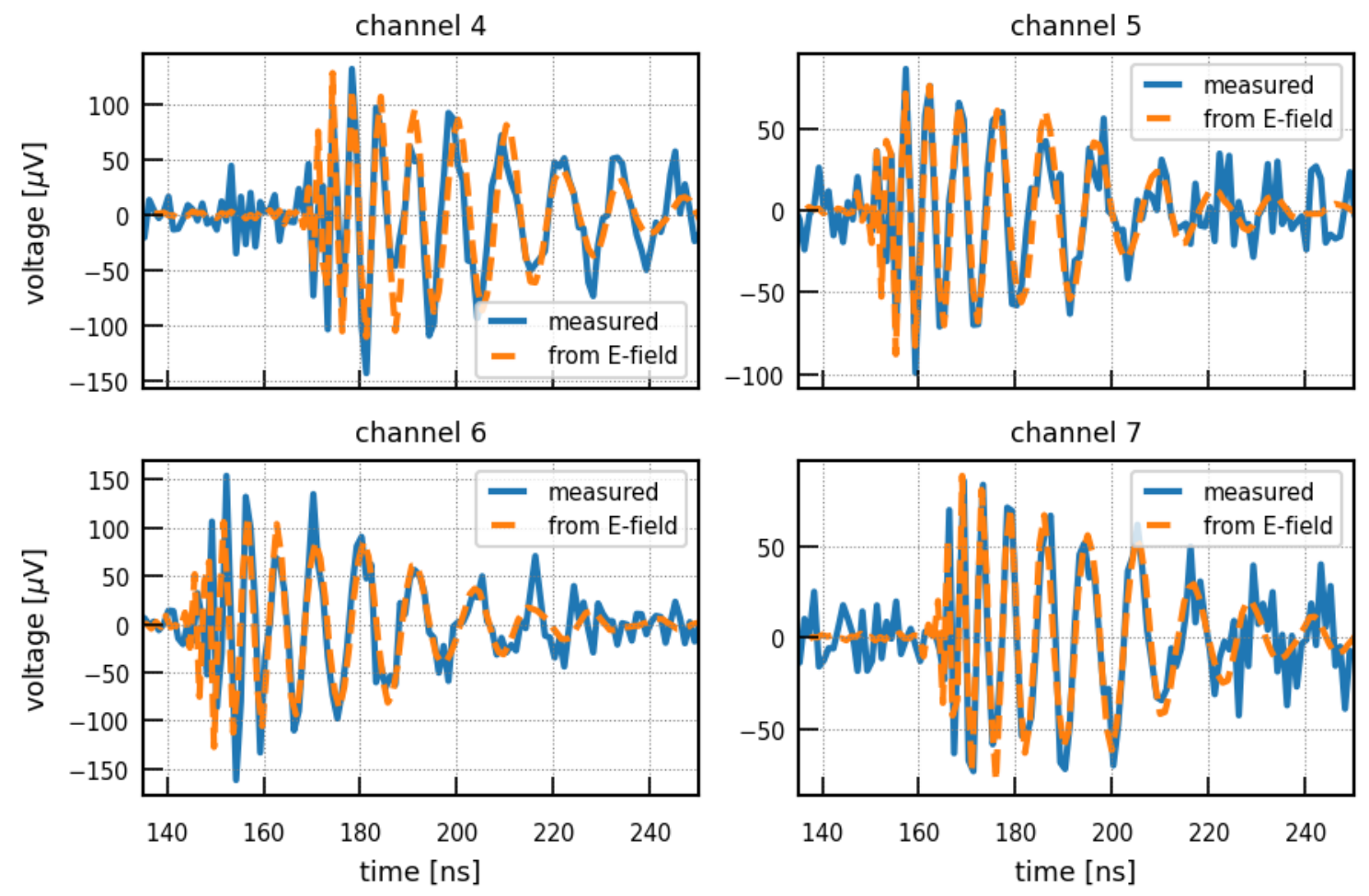

Figure 2: An example of electric field reconstruction using forward-folding technique. The blue curves represent the measured voltage traces and the orange dashed curves are the analytic solution of the forwardfolding technique.

add-on of Askaryan effect [8]. Therefore, a relatively accurate prediction of polarization can be made by $\vec{v} \times \vec{B}$, where $\vec{v}$ is the signal arrival direction and $\vec{B}$ is the local geomagnetic field vector. This prediction accounts only for the dominant geomagnetic effect but not for the subdominant Askaryan effect.

\subsection{Polarization error distribution}

Based on the reconstructed arrival direction, we can now predict the signal polarization and compare it to the reconstructed polarization. This allows us to asses the polarization resolution directly from measured cosmic rays. The resulting polarization error distribution is shown in Figure 3. We find a polarization uncertainty of $3.5^{\circ}$, which is a significant improvement compared to work in [3]. The distribution shows three clear outliers. We inspected these events in details and all of them have non-cosmic ray features which indicates that the set of cuts defined above can be further improved. With these three events removed, the polarization resolution would improve to $3.1^{\circ}$.

We performed the same reconstruction and analysis on a simulated data set consisting of CoREAS simulations where the events were re-weighted to correspond to the expected cosmic-ray flux. Each shower was reused several times with random core positions. The simulated observer position that is closest to the ARIANNA station was used and a full detector simulation was performed, including the addition of thermal noise. At this stage, the events looks like measured 


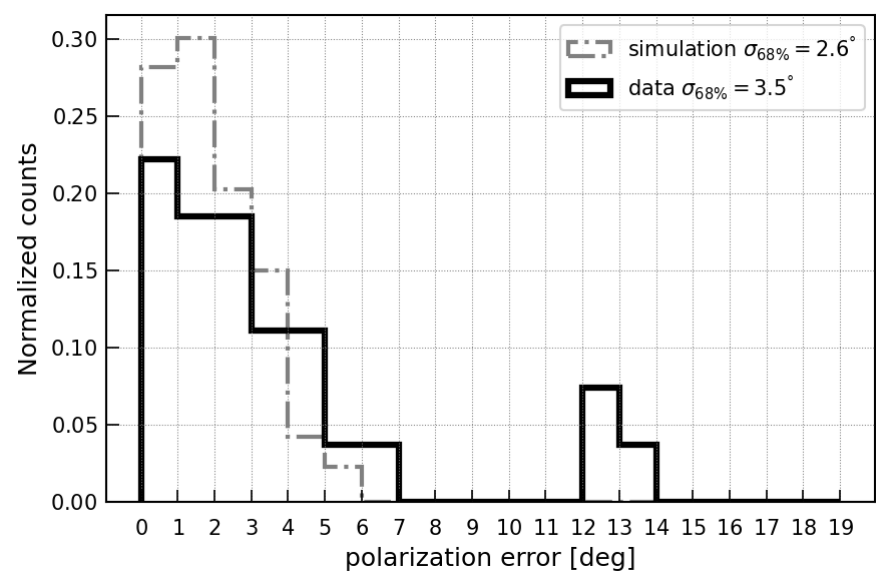

Figure 3: Solid histogram: polarization error of events in data with zenith $>40^{\circ}$. Dashed histogram: polarization error of events in simulation with zenith $>40^{\circ}$. The 3 outliers in data at $12^{\circ}$ have non-cosmic ray features. With them removed, $\sigma_{68 \%}$ would improve to $3.1^{\circ}$.

data and the signal polarization was reconstructed in the same way. We find a slightly better polarization resolution of $2.6^{\circ}$.

Moreover, according to simulation study, the error is dominated by uncertainties in polarization prediction rather than reconstruction. The predicted polarization only accounts for the contribution from the geomagnetic effect and not the sub-dominant Askaryan emission. This uncertainty was minor when the polarization resolution was large. However, the uncertainty due to not accounting for Askaryan effect is no longer negligible when we are able to measure polarization to a comparable accuracy. The influence of Askaryan effect on polarization is related to the station's position relative to the air shower axis. It is also inversely correlated to the angle between the local geomagnetic field vector and signal arrival direction vector, which reduces the strength of the geomagnetic emission. Since the local geomagnetic field is nearly vertical, the dependence on this angle is dominant by the dependence on zenith angle. Finally, the strength of the geomagnetic emission depends on the air density in which the shower develops and increases for thinner air densities [10], which reduces the influence of the Askaryan emission further for large zenith angles.

Therefore, we repeat the analysis with an increasing stringent cut on zenith angle. As shown in Figure 4, the polarization error is significantly reduced when we subselect events above a certain zenith threshold. This is because the strength of Askaryan effect decreases as zenith angle increases. With limited statistics, we are able to obtain a resolution of 1.3 degree ( $68 \%$ containment) when we subselect events with zenith $>70$ degree. It agrees with the simulation. More precisely, there are 3 events that have a reconstructed zenith angle larger than 70 degree. They have polarization errors of $0.6^{\circ}, 0.6^{\circ}$, and $1.94^{\circ}$.

\section{Conclusion}

We identified 148 cosmic rays from a 3-month data-taking period from Nov 2018 to Mar 2019. 27 events that have an SNR greater than 4.5 in all upward channels are selected for polarization study. We found that the reconstructed polarization agrees within $3.5^{\circ}$ with the predicted polarization 

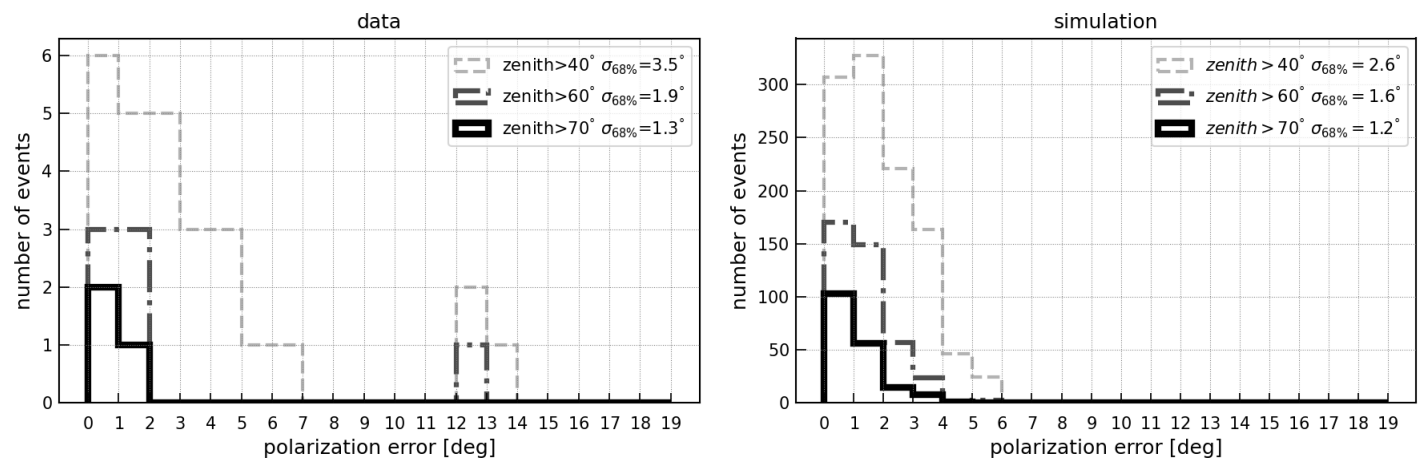

Figure 4: Polarization error distribution for events above certain zenith angles for data (left) and simulation (right). The error decreases significantly with increasing zenith angle. This is because The strength of Askaryan effect is related to zenith angle, and the polarization error is dominated by error in predicted polarization caused by Askaryan effect. $\sigma_{68 \%}$ is calculated from a linear interpolation to $68 \%$ of data. The 3 events in data with zenith $>70^{\circ}$ have polarization errors of $0.6^{\circ}, 0.6^{\circ}$, and $1.94^{\circ}$.

solely based on the geomagnetic effect and using the reconstructed air-shower direction. Moreover, we have found that the predicted polarization has a non-negligible contribution to the observed difference. The uncertainty in polarization prediction comes from the sub-dominant Askaryan radio emission in air showers, for which our prediction does not account. If we subselect events with larger zenith angles where the influence of the Askaryan effect is small, we find an improved agreement between reconstructed and expected polarization. In particular, the inferred resolution improves to 1.3 degree if we subselect events with a zenith angle larger than 70 degree.

\section{References}

[1] ARIANNA Collaboration, A. Anker et al. JCAP 03 (2020) 053.

[2] ARIANNA Collaboration, S. W. Barwick et al. Astropart. Phys. 90 (2017) 50-68.

[3] A. Nelles for the ARIANNA Collaboration PoS(ICRC2019)366 .

[4] C. Welling, C. Glaser, and A. Nelles JCAP 10 (2019) 075.

[5] C. Glaser for the ARIANNA collaboration PoS(ICRC2019)899.

[6] G. Gaswint, University of California, Irvine. PhD thesis, 2021.

[7] S. Barwick for the ARIANNA collaboration PoS ICRC2021 (these proceedings) 1151.

[8] T. Huege et al. AIP Conf. Proc. 1535 (2013) 128-132.

[9] C. Glaser et al. European Physical Journal C79 no. 6, (2019) 464.

[10] C. Glaser et al. JCAP 09 (2016) 24. 


\section{Full Authors List: ARIANNA Collaboration}

Astrid Anker ${ }^{1}$, Pierre Baldi ${ }^{2}$, Steven W. Barwick ${ }^{1}$, Jakob Beise ${ }^{3}$, Hans Bernhoff ${ }^{4}$, Dave Z. Besson ${ }^{5,6}$, Nils Bingefors ${ }^{3}$, Maddalena Cataldo $^{7}$, Pisin Chen ${ }^{8}$, Daniel García Fernández ${ }^{9}, 7$, Geoffrey Gaswint ${ }^{1}$, Christian Glaser ${ }^{3}$, Allan Hallgren ${ }^{3}$, Steffen Hallmann ${ }^{9}$, Jordan C. Hanson ${ }^{10}$, Spencer R. Klein ${ }^{11}$, Stuart A. Kleinfelder ${ }^{12}$, Robert Lahmann ${ }^{7,1}$, Jiayi Liu ${ }^{1}$, Mitchell Magnuson ${ }^{5}$, Stephen McAleer ${ }^{2}$, Zach Meyers ${ }^{9}$, Jiwoo $\mathrm{Nam}^{8}$, Anna Nelles ${ }^{9,7}$, Alexander Novikov ${ }^{5,6}$, Manuel P. Paul ${ }^{1}$, Christopher Persichilli ${ }^{1}$, Ilse Plaisier ${ }^{9,7}$, Lilly Pyras $^{9}$, Ryan Rice-Smith ${ }^{1}$, Joulien Tatar ${ }^{13}$, Shih-Hao Wang ${ }^{8}$, Christoph Welling ${ }^{9,7}$, Leshan Zhao ${ }^{1}$

${ }^{1}$ Department of Physics and Astronomy, University of California, Irvine, CA 92697, USA

${ }^{2}$ Department of Information and Computer Science, University of California, Irvine, CA 92697, USA.

${ }^{3}$ Uppsala University Department of Physics and Astronomy, Uppsala SE-752 37, Sweden.

${ }^{4}$ Uppsala University Department of Engineering Sciences, Division of Electricity, Uppsala SE-752 37, Sweden.

${ }^{5}$ Department of Physics and Astronomy, University of Kansas, Lawrence, KS 66045, USA.

${ }^{6}$ National Research Nuclear University MEPhI (Moscow Engineering Physics Institute), Moscow 115409, Russia.

${ }^{7}$ ECAP, Friedrich-Alexander Universität Erlangen-Nürnberg, 91058 Erlangen, Germany.

${ }^{8}$ Department of Physics and Leung Center for Cosmology and Particle Astrophysics, National Taiwan University, Taipei 10617, Taiwan. ${ }^{9}$ DESY, 15738 Zeuthen, Germany.

${ }^{10}$ Whittier College Department of Physics, Whittier, CA 90602, USA.

${ }^{11}$ Lawrence Berkeley National Laboratory, Berkeley, CA 94720, USA.

${ }^{12}$ Department of Electrical Engineering and Computer Science, University of California, Irvine, CA 92697, USA.

${ }^{13}$ Research Cyberinfrastructure Center, University of California, Irvine, CA 92697, USA.

\section{Acknowledgement}

We are grateful to the U.S. National Science Foundation-Office of Polar Programs, the U.S. National Science Foundation-Physics Division (grant NSF-1607719) for supporting the ARIANNA array at Moore's Bay. Without the invaluable contributions of the people at McMurdo Station, the ARIANNA stations would have never been built. We acknowledge funding from the German research foundation (DFG) under grants GL 914/1-1 and NE 2031/2-1m, the Taiwan Ministry of Science and Technology, the Swedish Government strategic program Stand Up for Energy, MEPhI Academic Excellence Project (Contract No. 02.a03.21.0005) and the Megagrant 2013 program of Russia, via agreement 14.12.31.0006 from 24.06.2013. This work was supported by National Science Foundation Grant DGE-1633631. 